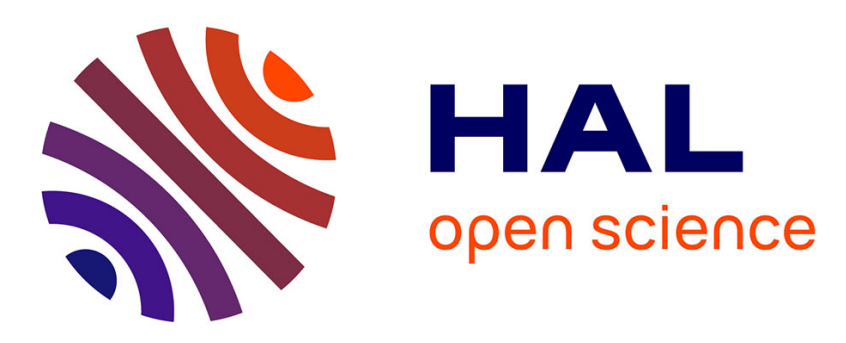

\title{
Analytical energy-efficient planning of 5G cloud radio access network
}

\author{
Riccardo Bassoli, Marco Di Renzo, Fabrizio Granelli
}

\section{To cite this version:}

Riccardo Bassoli, Marco Di Renzo, Fabrizio Granelli. Analytical energy-efficient planning of 5G cloud radio access network. IEEE International Conference on Communications (ICC 2017), May 2017, Paris, France. 10.1109/ICC.2017.7996871 . hal-01880000

\section{HAL Id: hal-01880000 https://hal.science/hal-01880000}

Submitted on 15 Jul 2020

HAL is a multi-disciplinary open access archive for the deposit and dissemination of scientific research documents, whether they are published or not. The documents may come from teaching and research institutions in France or abroad, or from public or private research centers.
L'archive ouverte pluridisciplinaire HAL, est destinée au dépôt et à la diffusion de documents scientifiques de niveau recherche, publiés ou non, émanant des établissements d'enseignement et de recherche français ou étrangers, des laboratoires publics ou privés. 


\title{
Analytical Energy-Efficient Planning of 5G Cloud Radio Access Network
}

\author{
Riccardo Bassoli ${ }^{1}$, Marco Di Renzo ${ }^{2}$, Fabrizio Granelli ${ }^{1}$ \\ ${ }^{1}$ University of Trento, Trento, Italy \\ ${ }^{2}$ Paris-Saclay University, Paris, France \\ $\{$ riccardo.bassoli,fabrizio.granelli\}@unitn.it \\ $\{$ marco.direnzo\}@12s.centralesupelec.fr
}

\begin{abstract}
5G wireless communication envisions unprecedented changes in current mobile networks in order to guarantee significant higher performance mainly in terms of data rates, latencies and efficiency. This paper provides an analytical model based on stochastic geometry, in order to address the planning and the dimensioning of 5G Cloud RAN. The results shows the requirements in terms of number of virtual baseband units, and the energy gain achieved by virtualisation both in micro-cell and pico-cell scenarios. Thus, the proposed methodology represents a step forward to analyse and compare traditional RAN design with the emerging Cloud RAN paradigm.
\end{abstract}

\section{Index Terms}

5G, virtualisation, cloud computing, consolidation, stochastic geometry, green networks.

\section{INTRODUCTION}

The vision of future 5G technologies [1] requires relevant increase in performance, while changing the overall network and architecture. In particular, the requirements mainly affect data rates, round-trip latency, bandwidth in unit area, perceived available connectivity, number of connected mobile terminals (MTs) and energy usage.

Regarding that, virtualisation, Software Defined Networking (SDN) and Cloud Radio Access Network (RAN) [2], [3] have been recommended as the main technologies in order to solve the major problems related to increasing data rates, reducing latencies and energy efficiency.

The idea behind Cloud RAN is to enable higher flexibility in management and configuration of the RAN section of a cellular network by detaching base-band processing functionalities from standard access points (APs) or eNodeBs, and moving them in convenient locations where shared processing facilities will be available. In this way, eNodeBs can become less complex and less energy demanding (in some cases 
as simple as Remote Radio Heads - RRHs), and their processing functionalities can become virtualised network functions named virtual Base-Band Units (v-BBUs). This allocation will provide more flexibility and efficiency as the position and processing power allocated to each v-BBU could be defined based on the current load of each RRH and the operating context. Moreover, the resources for v-BBU can be easily migrated by providing cost-effective consolidation of co-located virtual entities.

Among the benefits of virtualisation there is also the possibility to achieve further energy and resource utilisation efficiency by exploiting the concept of 'consolidation'. Consolidation of virtual machines [4] is a technology to reduce energy consumption of data centres. The locations of virtual machines are continuously re-optimised in response to its resource requirements. The idea behind it is to monitor resources of VMs in order to optimise the location of VMs. In particular, the system groups VMs inside the minimum number of servers and keeps off the unused ones. In case of overloading, the system manage the unused servers to turn them on and to relocate VMs.

It is not straightforward to estimate this component, but considering available works [4] we assume that consolidation is able to provided a further $20 \%$ reduction in energy consumption and that the power consumption incurred by live migration itself can be considered negligible.

Quantitative estimations of the performance of Cloud RAN architecture or the effectiveness in terms of energy and costs of such paradigm are not yet addressed in the literature. The main scope of this work is to provide an analytical model to study and to design 5G Cloud RAN, based on stochastic geometry. Stochastic geometry is a new approach based on the theory of point processes (PP) for systemlevel modelling, performance evaluation and optimisation of future cellular networks. In particular, the locations of cellular APs are not assumed to be regularly deployed, but they are randomly distributed according to a PP. This approach, due to its mathematical flexibility for modelling heterogeneous ultradense cellular deployments, is today considered the most adequate for designing and optimising future networks [5]. Our initial model has the capability to estimate the average number of v-BBUs required by the network, and thus the energy consumption achievable by deploying virtualisation. Currently, to the best of authors' knowledge, the only quantitative analysis of the number of required v-BBUs in a $5 \mathrm{G}$ scenario is presented in [6].

The paper is organised as follows. Section II describes the system model and the theory of stochastic geometry used in the work. Next, section III provides the data of the scenario, and calculates how the number of v-BBUs and the energy consumption changes during the day: in particular, the analytical evaluation compares three technologies such as 4G/LTE, Cloud RAN and Cloud RAN with consolidation. 


\section{System MODEL}

Let's consider a cellular 4G/LTE network with single-input single-output (SISO) access points (AP), distributed on an Euclidean plane $\mathbb{R}^{2}$ according to a two-dimensional homogeneous Poisson point process (PPP) $\Phi_{A P}$ of intensity $\lambda_{A P}$. The mobile terminals (MTs) are also distributed according to a homogeneous PPP $\Phi_{M T}$ of intensity $\lambda_{M T}$, which is independent of $\Phi_{A P}$. In the network, each MT is associated to the nearest AP.

Base-band units (BBUs) are units that perform baseband (PHY/MAC) processing. In the LTE standard, this equipment needs to be installed within each eNodeB, close to the Remote Radio unit (RRU). However, next generation $5 \mathrm{G}$ technologies aim to virtualise processing tasks performed by current BBUs, and to implement them in the core network as virtual network functions (v-BBUs). The application of a stochastic-geometric model to design 5G Cloud RAN is fundamental since the dynamic/flexible allocation of resources of BBUs can improve the efficiency of the current cellular networks.

By using the statistics elaborated in [7], it is possible to obtain the average percentage of active users, connected to the cellular network in a dense urban scenario. The considered urban scenario for our model is the city of London. A realistic urban density of APs is approximately $\lambda_{A P}=80 \mathrm{AP} / \mathrm{km}^{2}$ [8], and the users' density can be set to $\lambda_{M N}=10 \lambda_{A P} \mathrm{MT} / \mathrm{km}^{2}$ [8]. Given these premises, figure 1 clearly depicts the average variation of density of active mobile users $\left(\lambda_{M T}\right)$ according to the hours of the day.

By considering the stochastic-geometric model studied by the authors in [9], under the above assumptions it is possible to derive the random variable $N_{M T}$, which denotes the number of MTs in a Voronoi cell associated to a randomly chosen AP. Hence, the probability mass function (pmf) of $N_{M T}$ is

$$
P\left[N_{M T}=n\right]=\frac{3.5^{3.5} \Gamma(n+3.5)\left(\lambda_{M T} / \lambda_{A P}\right)^{n}}{\Gamma(3.5) n !\left(\lambda_{M T} / \lambda_{A P}+3.5\right)^{n+3.5}}
$$

where $\Gamma(x)$ represents the gamma function. It is assumed that each MT can be served in a single resource block at a given time. Next, the probability to have an inactive AP can be expressed as

$$
p_{\text {off }}=\left(1+3.5^{-1} \lambda_{M T} / \lambda_{A P}\right)^{-3.5}
$$

Finally, the paper considers that the cloud servers have enough processing power, so the computational capabilities of v-BBUs are the same as the corresponding BBUs.

\section{RESULTS AND DisCUSSIONS}

This section exploits the model based on stochastic geometry in order to calculate the variation of the average number of v-BBUs of the network during the day. Based on such figures, evaluation of the energy consumption of micro-cell and pico-cell scenarios with v-BBUs is provided and compared with current 4G/LTE networks. 


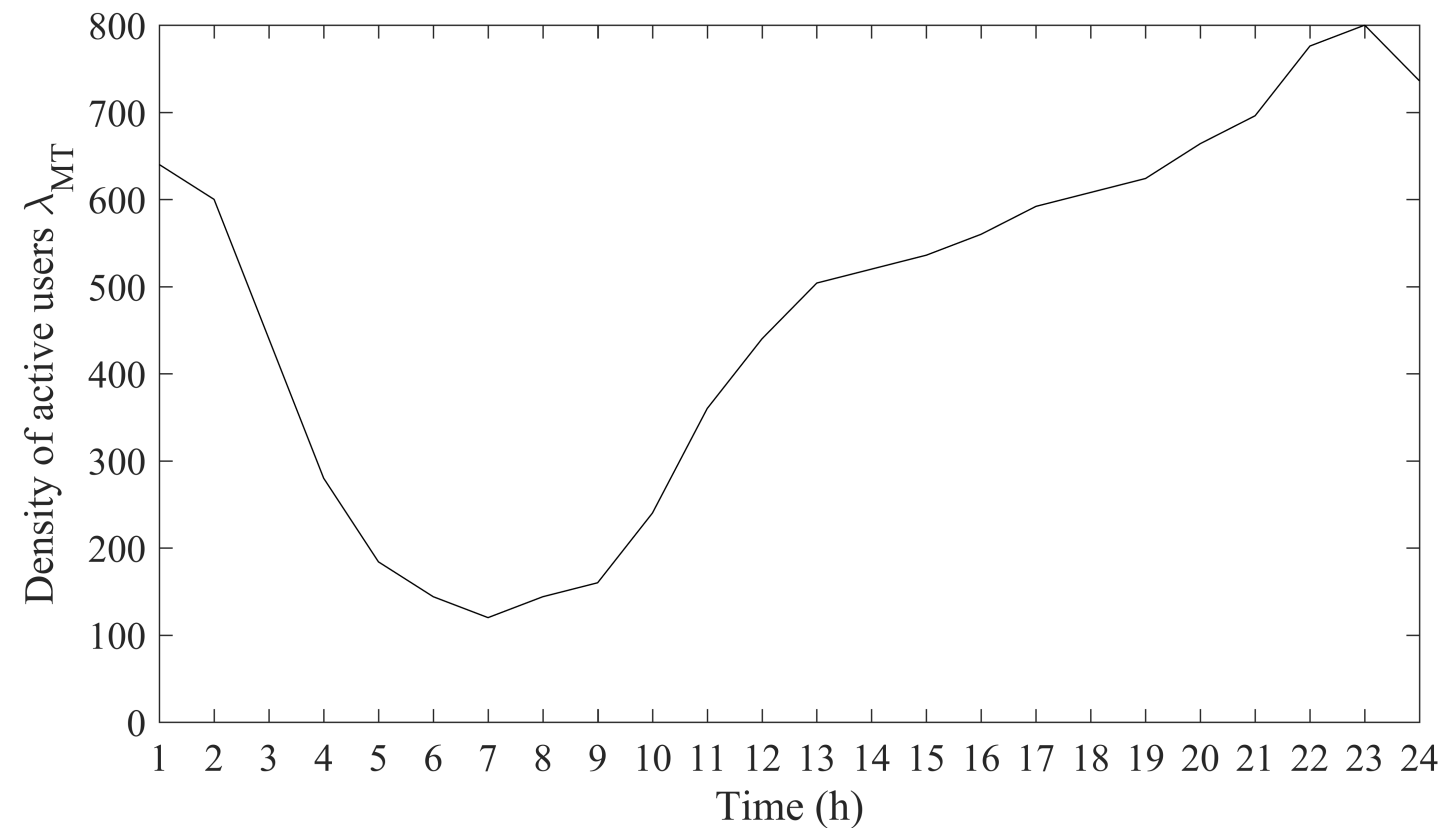

Fig. 1. Average variation of density of active mobile users $\left(\lambda_{M T}\right)$ according to the hours of the day.

\section{A. Estimation of $v$-BBUs}

In order to provide a realistic figure of the computational power of the BBUs, the technical specifications for the Huawei model eBBU530 [10] are used. Currently most of mobile data traffic is dedicated to social networks, high-quality video streaming, and VoIP. In future 5G networks, this trend will be even more evident. Given that, each MT keeps an active data rate of 2.5 Mbps. Thus, a single BBU can support a maximum number of MTs, which is approximately modelled by the parameter $\eta=50$.

The analysis based on stochastic geometry uses the expected values of $N_{M T}$ in order to evaluate the mean value of active v-BBUs. Hence, it is possible to estimate the average number of active v-BBUs that the network requires, and the average number of Voronoi cells that a single v-BBU has to serve. Figure 2 represents the number of v-BBUs required by the scenarios with micro and pico cells, versus the hours of the day.

\section{B. Analysis of Energy Consumption}

The application of virtualisation allows the definition of a dynamic scenario, in which v-BBUs are activated according to the needs of traffic. This does not happen in current 4G/LTE, where each active base station always hosts an active BBU. This means that 4G/LTE networks keep on $\lambda_{A P}=80 \mathrm{AP} / \mathrm{km}^{2}$ and $\lambda_{A P}=240 \mathrm{AP} / \mathrm{km}^{2}$ BBUs for micro and pico cells, respectively. In current mobile networks, $p_{o f f}$ 


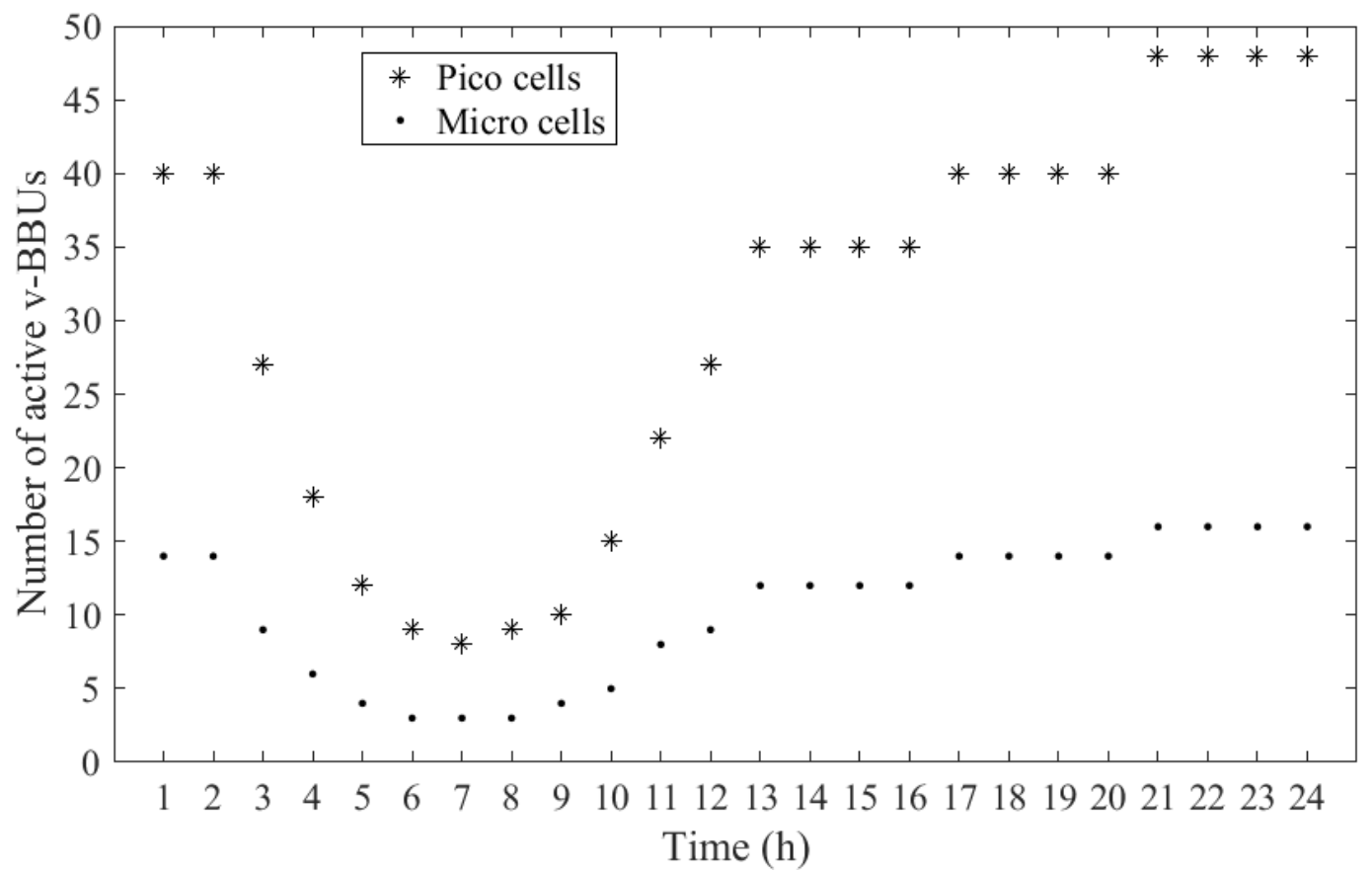

Fig. 2. Average number of active v-BBUs in micro- and pico-cell scenarios during the time of the day.

can affect the number of active BBUs: in fact, when an AP is inactive, its BBU is off. The evaluation of $p_{\text {off }}$ shows that micro-cell APs are always active with high probability, while around $10 \%$ pico-cell APs can be inactive.

The energy consumption of a BBU can be estimated as $27.3 \mathrm{~W}$ for micro cells and $3 \mathrm{~W}$ for pico cells [7]. Regarding the v-BBUs, we considered the same energy consumption of BBUs, plus the energy consumed to cool the sites where the devices are located [7].

Figure 3 shows the daily profile of total energy consumption due to BBUs/v-BBUs in micro-cell scenario for the three considered technologies. The value of $p_{\text {off }}$ is negligible except during early morning hours, when less users are connected: hence, the presence of less active APs reduces the total energy consumption in all the three scenarios. Nevertheless, if we deploy virtualisation by simply activating v-BBUs according to the needs, the network can experience a significant energy saving for BBUs supply. Moreover, this energy saving can be further increased by taking advantage of virtual machine consolidation, a methodology employed in data centres to group several virtual machines and reduce the number of active hosts.

Figure 4 depicts how the total energy consumption due to BBUs/v-BBUs in pico-cell scenario changes during the day. In this case, $p_{\text {off }}$ also reduces the energy consumption of 4G/LTE in daylight times since 


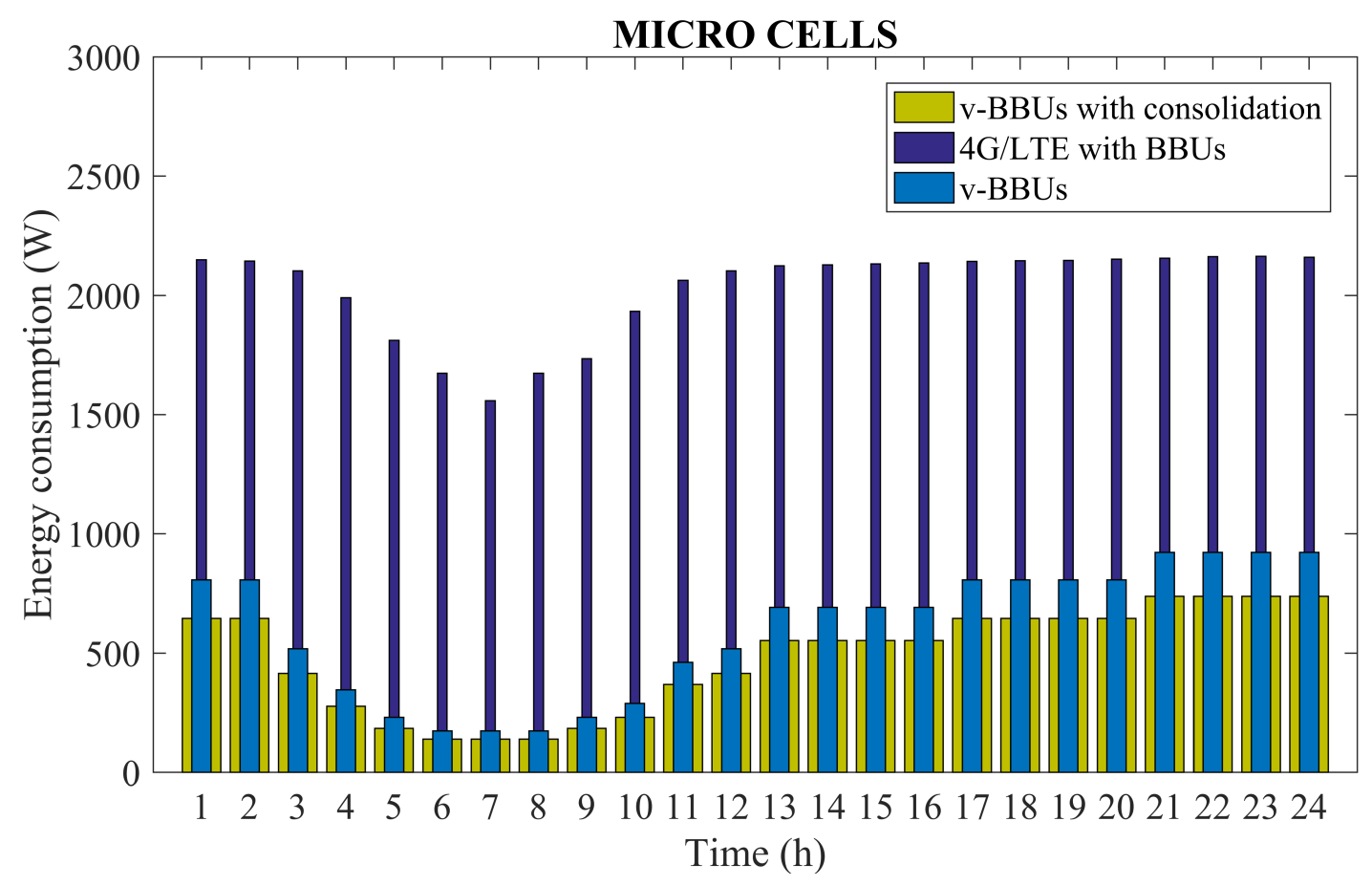

Fig. 3. Total energy consumed by BBUs/v-BBUs in cloud RAN (with v-BBUs) and in current 4G/LTE scenario (with BBUs) with micro cells. The comparison includes the possibility of applying virtual machines' consolidation for v-BBUs.

APs, and thus their BBUs, can be inactive with significant probability. By comparing figure 4 and figure 3 it is clear that pico cells can further increase energy saving. Furthermore, the application of consolidation can make virtualisation more efficient.

Figure 5 plots the energy gains provided during the day by using the Cloud RAN paradigm (with and without consolidation) instead of legacy 4G/LTE technologies. These gains are only referred to BBUs/vBBUs. It is possible to notice that greater gains are achieved during early-morning hours, when there are less users connected. Moreover, the application of v-BBUs can provides gains greater than $55 \%$ along all the day. The comparison of micro-cell and pico-cell scenarios shows that the latter can achieve higher energy saving, especially when more users are connected to the network. Finally, the gain provided by the consolidation technique increases when the network has to serve more users, thus when more v-BBUs are on.

Finally, figure 6 and figure 7 depict the total energy consumed by the network for the three technologies. In this case, the calculation includes not only the cost of BBUs/v-BBUs but also the energy required to maintain active the entire APs that are ON. According to the energy model described in [7], the additional costs to add are the ones due to power amplifier (PA), Radio Frequency (RF) small-signal transceiver, 


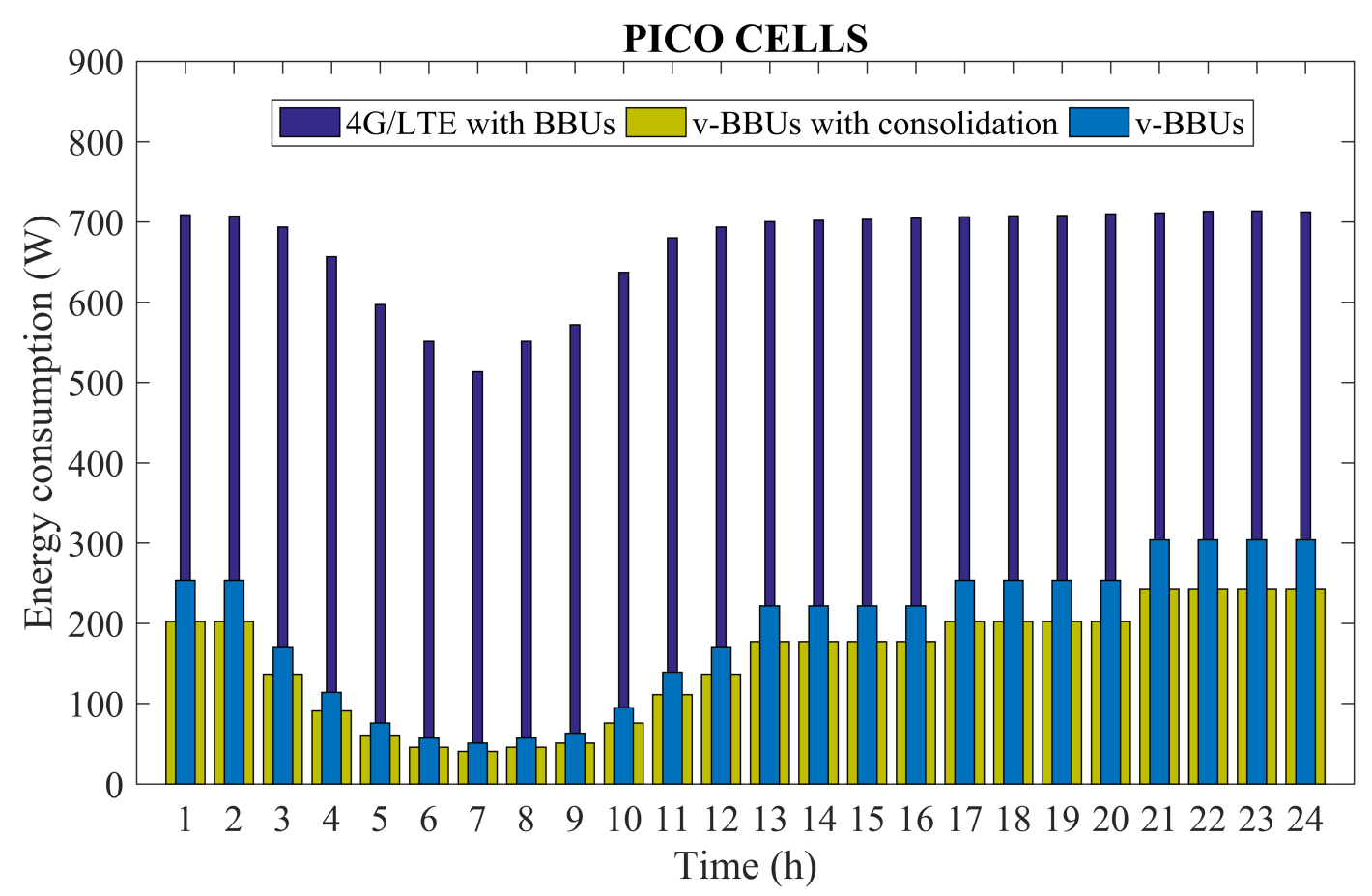

Fig. 4. Total energy consumed by BBUs/v-BBUs in Cloud RAN (with v-BBUs) and in current 4G/LTE scenario (with BBUs) with pico cells. The comparison includes the possibility of applying virtual machines' consolidation for v-BBUs.

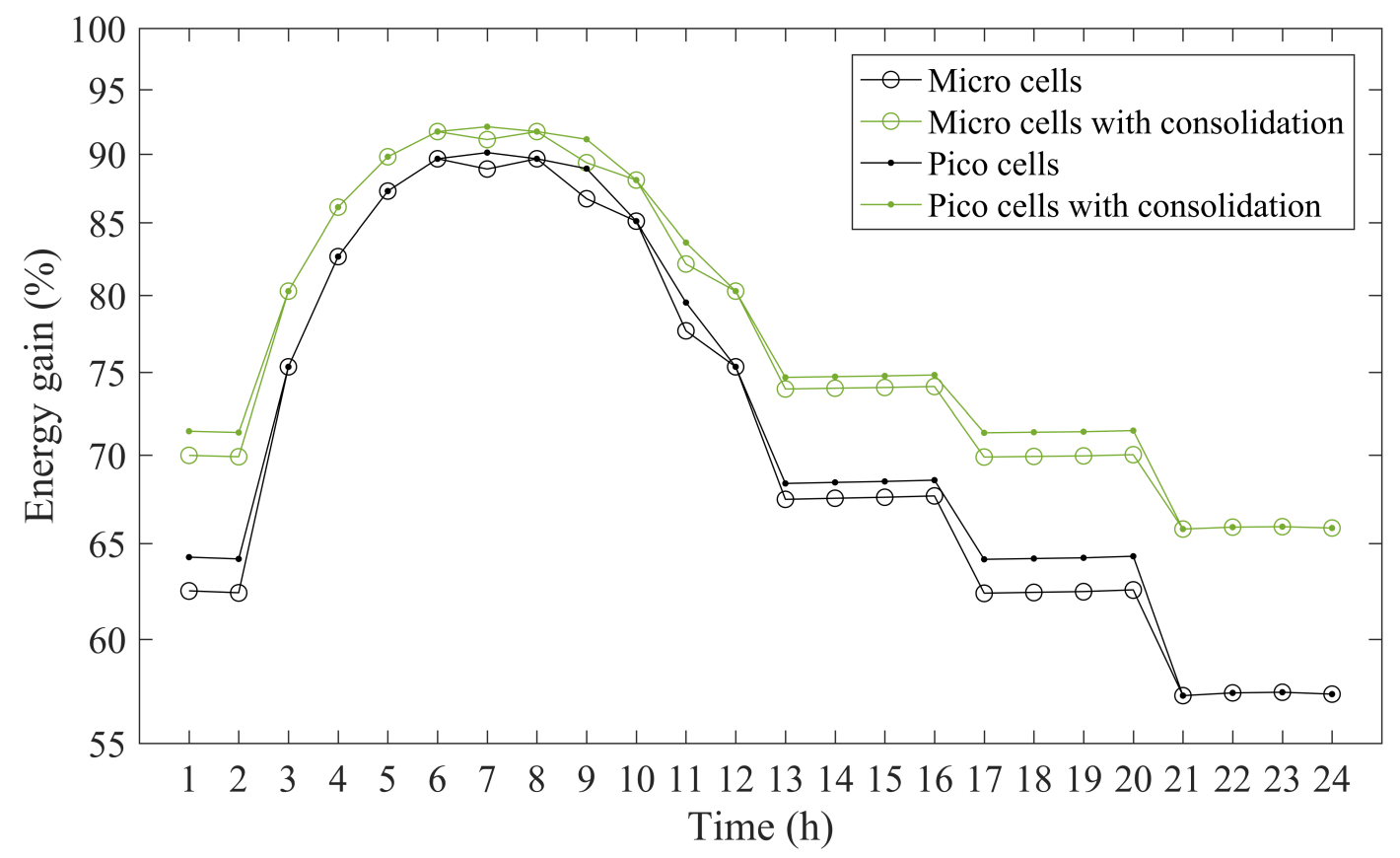

Fig. 5. Energy gain obtained by using micro and pico cells in Cloud RAN (with v-BBUs) in respect of current 4G/LTE scenario (with BBUs). The gain is also calculated considering the possibility of applying virtual machines' consolidation. 


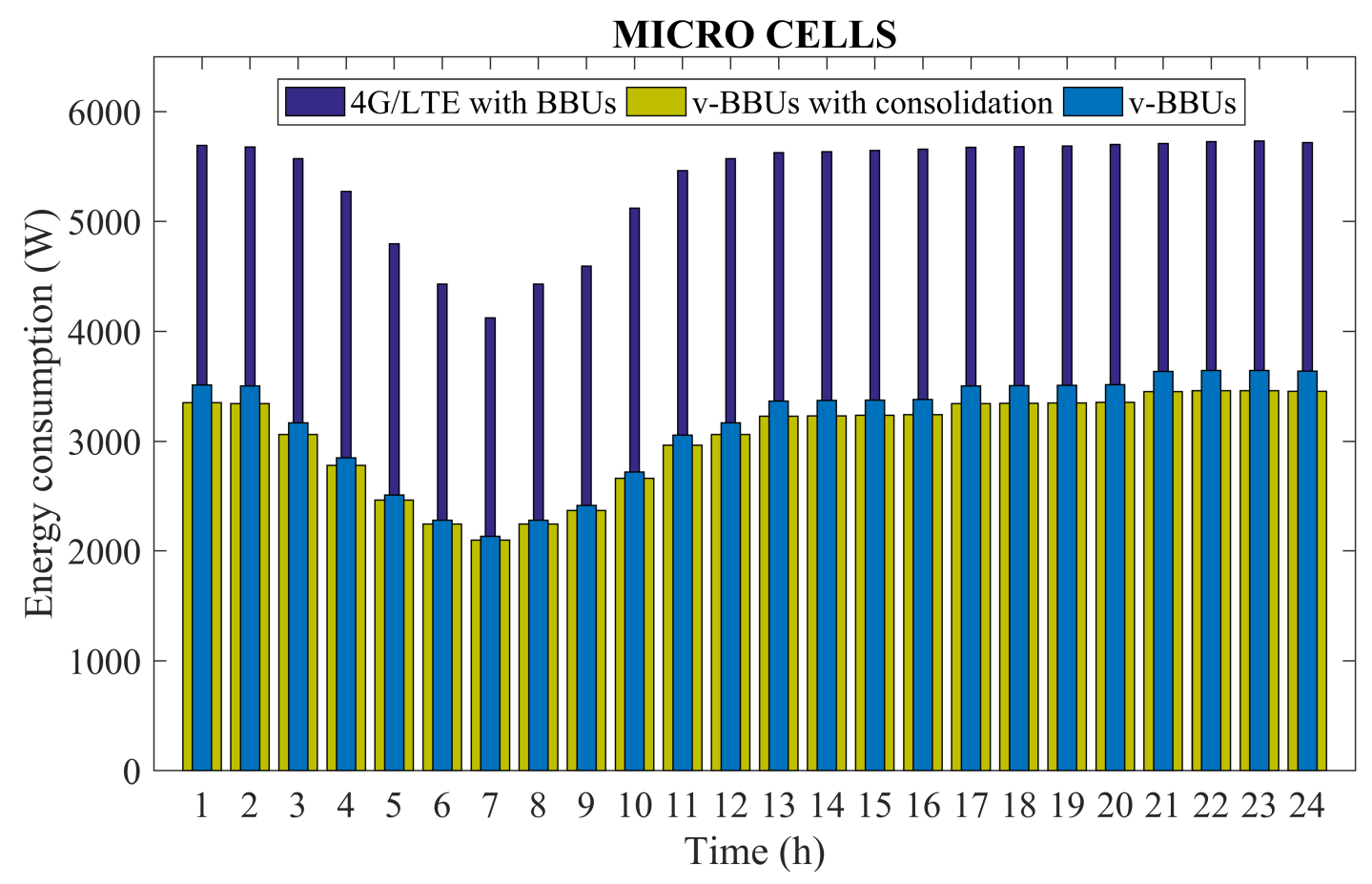

Fig. 6. Total energy consumed by using micro cells in Cloud RAN (with v-BBUs and consolidation) and in current 4G/LTE scenario (with BBUs). The consumptions include the energy required for entire AP activity.

DC-DC power supply, and an AC-DC unit for connection to the electrical power grid.

By taking into account the energy consumption of the entire RAN (APs + BBUs), the gains provided by Cloud RAN change as follows. In case of micro cells, they vary between $38 \%$ and $48 \%$ without consolidation, and between $41 \%$ and $48 \%$ with consolidation, while with pico cells, they change between $42 \%$ and $55 \%$ without consolidation, and between $48 \%$ and $56 \%$ with consolidation.

\section{CONCLUSIONS}

The paper has described an analytical model to analyse and compare traditional RAN with Cloud RAN architectures for future 5G networks. A model based on stochastic geometry has been used to calculate the energy consumed by current 4G/LTE RAN and Cloud RAN in the same setup. In particular, the energy consumption has been evaluated according to the variation of connected mobile terminals during the day. The results show that Cloud RAN and virtualisation can provide a significant energy saving, greater than $38 \%$ for the whole network. Moreover, the energy gains of virtualisation can be further increased by deploying efficient techniques like virtual machine consolidation.

Future works include: the extension of the stochastic-geometric model to scenarios in which micro and pico cells coexist in the same network, the evaluation of energy consumption by including core network 


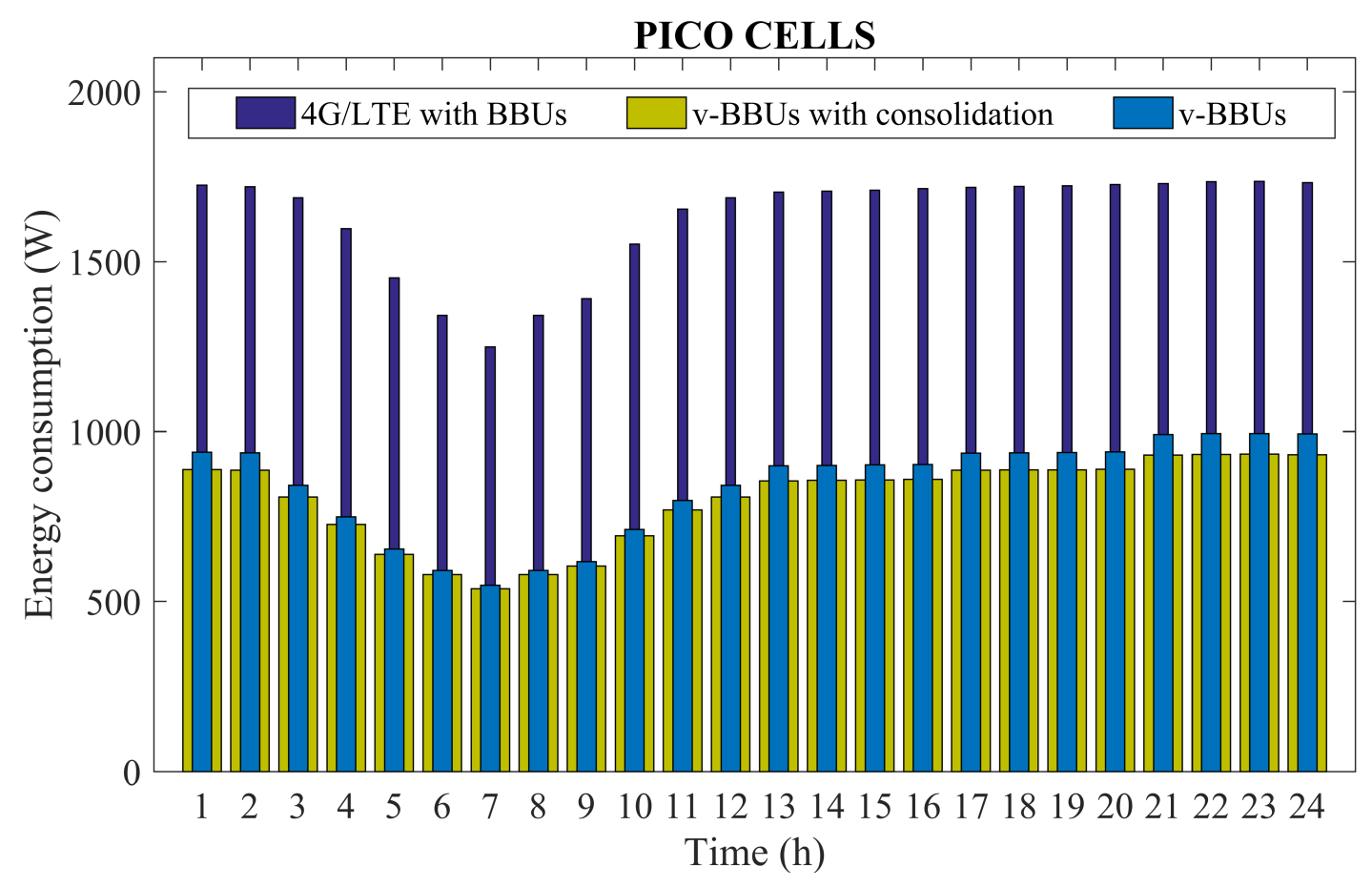

Fig. 7. Total energy consumed by using pico cells in Cloud RAN (with v-BBUs and consolidation) and in current 4G/LTE scenario (with BBUs). The consumptions include the energy required for entire AP activity.

contribution, and the estimation and optimisation of communication latency.

\section{REFERENCES}

[1] M. Agiwal, A. Roy, and N. Saxena, "Next generation 5G wireless networks: A comprehensive survey," IEEE Communications Surveys Tutorials, vol. 18, no. 3, pp. 1617-1655, thirdquarter 2016.

[2] Ericsson, “Cloud RAN,” White Paper, Sep. 2015, uen 284 23-3271.

[3] Fujitsu, "The benefits of Cloud-RAN architecture in mobile network expansion," White Paper, 2015.

[4] T. Hirofuchi, H. Nakada, S. Itoh, and S. Sekiguchi, "Making VM consolidation more energy-efficient by postcopy live migration," in The 2nd International Conference on Cloud Computing, GRIDs, and Virtualization, Jul. 2011.

[5] M. Haenggi, Stochastic Geometry for Wireless Networks, 1st ed. New York, NY, USA: Cambridge University Press, 2012.

[6] S. Abdelwahab, B. Hamdaoui, M. Guizani, and T. Znati, "Network function virtualization in 5G," IEEE Communications Magazine, vol. 54, no. 4, pp. 84-91, Apr. 2016.

[7] G. Auer, V. Giannini, I. Godor, P. Skillermark, M. Olsson, M. A. Imran, D. Sabella, M. J. Gonzalez, C. Desset, and O. Blume, "Cellular energy efficiency evaluation framework," in IEEE 73rd Vehicular Technology Conference (VTC Spring), May 2011, pp. 1-6.

[8] W. Lu and M. D. Renzo, "Stochastic geometry modeling of cellular networks: Analysis, simulation and experimental validation," in ACM International Conference on Modeling, Analysis and Simulation of Wireless and Mobile Systems, Cancun, Mexico, Nov. 2015. 
[9] M. D. Renzo, W. Lu, and P. Guan, "The intensity matching approach: A tractable stochastic geometry approximation to system-level analysis of cellular networks," vol. abs/1604.02683. [Online]. Available: http://arxiv.org/abs/1604.02683

[10] (2012) eWBB TDD 3.0 DBS3900 - product description. [Online]. Available: http://www.huawei.com/ilink/cnenterprise/ download/HW_203790 